\title{
The "behind-the-knee" test for menstrual tampon irritation
}

\author{
Miranda A. Farage ${ }^{1 *}$, Kenneth W. Miller ${ }^{1}$, William J. Ledger ${ }^{2}$ \\ ${ }^{1}$ Feminine Clinical Sciences, The Procter \& Gamble Company, Cincinnati, USA \\ ${ }^{2}$ Department of Obstetrics and Gynecology, The New York-Presbyterian Hospital, Weill Medical College, Cornell University, New \\ York, USA \\ Email: farage.m@pg.com
}

Received 2 November 2011; revised 4 December 2011; accepted 31 December 2011

\section{ABSTRACT}

Colposcopic inspection of the vagina is a routine component of the safety assessment of intravaginal products. However, colposcopic findings occur frequently in healthy women, raising questions about their relevance to intravaginal product safety. Practical disadvantages limit the utility of colposcopy for evaluating menstrual tampons, among them the presence of background microtrauma, the inability to assess effects during menstruation, and, importantly, the question of whether post-hoc assessments are sufficiently sensitive to detect small inflammatory changes. The Behind-the-knee (BTK) test is an alternative for evaluating inflammatory and tissue dryness effects of physical articles by their repeated application to the popliteal fossa under an elastic bandage. It enables concurrent parallel comparisons of experimental and control articles over time and substantially increases the sensitivity of detecting small changes in tissue inflammation. With this protocol, uncompressed experimental and control tampons yielded comparable relative and absolute erythema scores (after overnight recovery) as did colposcopic assessment of the lower genital tract $\mathbf{3}$ to $\mathbf{4 8}$ hours after menstrual use. Scoring erythema in the BTK test immediately after product removal increased the level of visually discernible inflammation 6-fold. In a study of commercial menstrual pads, subclinical inflammation visualized with cross-polarized light correlated with the frequency of subjective reports of discomfort during the test and discriminated the relative tolerability of the two products determined by market surveillance, providing added confidence in the predictive value of the test. We believe the BTK test to be a valuable alternative to colposcopy for assessing inflammation and dryness associated with menstrual tampons.

Keywords: Colposcopy; Behind-the-Knee; Menstrual

\footnotetext{
"Corresponding author.
}

Tampons

\section{INTRODUCTION}

Colposcopy (colpo: vagina; scope: to look) was first described in 1925 as a screening tool for cervical cancer but was later supplanted for primary screening by the Papanicolaou test (Pap smear). In clinical practice, colposcopy is performed on women with abnormal cytological screening to examine potentially precancerous or cancerous lesions. In the wake of studies from the 1990s that identified unexpected epithelial disruption from intravaginal contraceptive products, greater emphasis was placed on investigating potential damage (e.g. epithelial or blood vessel disruption) that might increase the risk of STI or HIV transmission [1]. Thus, colposcopy entered the standard arsenal for the safety assessment of intravaginal products (including tampons) mandated by the US Food and Drug Administration regulatory approval process.

With broad scale use of colposcopy in the product research setting, its limitations have become more apparent [2]. On the most fundamental level, we do not fully understand the natural history and clinical significance of minor alterations to the vaginal epithelium, many of which occur absent product use. For example, erythema and petechiae of the cervix and vagina were found at baseline colposcopic evaluation of about one-third of women entering product use studies [3] and many superficial alterations were observed over four to six months of colposcopic inspection of the vaginal epithelium of healthy women [4]. Advancing age [3], tampon use [4], smoking [4], and intercourse in the prior 72 hours [4,5] contribute to vaginal microtrauma; these and other likely confounding factors (the menstrual cycle, exogenous hormones, barrier contraceptive use) create background "noise" of uncertain clinical significance $[2,6]$ that may make it difficult to assess the impact of changes in products designed to be minimally irritating, such as menstrual tampons. 


\section{DISCUSSION}

Especially relevant to tampon safety is the ability to discern possible chemical and physical irritation to the mucosa or tissue dryness from absorptive effects. Although the colposcopic light source and magnification aid in visualizing frank epithelial or vascular disruption [6], light reflected off the vaginal mucosal surface limits visibility of the subepithelial vasculature, a necessary step to assess the extent and depth of any inflammation noted. We found that colposcopy was no more sensitive than the naked eye for discerning erythema, inflammation, and tissue dryness in the lower genital tract of women with and without vulvovaginal symptoms; a cross-polarized light system that enabled subsurface visualization was more sensitive at detecting these endpoints, regardless of the presence of symptoms [7].

The safety assessment ideally will compare the experimental product under development with a relevant control, such as a similar product with regulatory approval and a well-established safety profile. Tampon use studies typically employ a crossover design to effect the comparison, with attendant shortcomings such as assessment of the test and control products in different cycles and the need for a washout period between crossover phases (the interval until the next menstrual period).

A significant limitation in tampon development research is that colposcopy is not employed during menstruation but performed 3 to 48 hours after removal of the last tampon used. This is OK to trying to determine the plot of a novel by reading the last chapter: the development and resolution of tissue effects during tampon wear is not discerned. Tissue alterations upon examination may represent residual product effects, variables unrelated to product use, or normal epithelial turnover and shedding.

Other practical limitations affect the use of colposcopy in the research setting. Colposcopy is an art developed by trained, experienced personnel: consequently, considerable interindividual variability exists between observers depending on their level of experience and the severity of the conditions examined [6,8]. Though the examination is not lengthy, some potential study subjects view it as invasive and discomfiting; this reluctance may promote recruitment of a self-selected sample that may or may not be representative of the general population.

An alternative approach for examining the epithelial effects of tampons in research and development would allow parallel comparison of the test and control products, have the requisite sensitivity to discern small changes in inflammation, allow tissue effects to be monitored over time, be less subject to confounding physiological or lifestyle factors, be able to be performed during menstruation, limit observer bias, and be non-invasive and minimally disruptive to human volunteers. However, the ideal alternative system does not yet exist: animal mod- els of the vagina are inadequate morphologically and physiologically, and cell culture systems, while more representative and potentially useful, are surrogate systems that rely on markers of inflammation. Monitoring inflammatory markers in clinical trials of intravaginal products is the subject of ongoing research [9].

In recent years, a new test model, now an ASTM standard protocol F2808, dubbed the "Behind-the-knee" (BTK) test [10], has shown potential utility for assessing inflammation and tissue dryness caused by articles that involve prolonged tissue contact, such as menstrual pads and tampons. In this simple protocol, the article is applied to the popliteal fossa under an elastic bandage and held in place for 6 hours a day over a period of up to 5 days. Tissue erythema and dryness are scored immediately after removal of the article, and again before its next application (a time frame that represents a recovery period of about 18 hours). Unlike traditional patch tests, this protocol both intensifies the degree of product contact and occlusion and introduces the element of friction due to movement of the joint to which the article has been applied. This leads to heightened sensitivity to both chemical and physical effects: notably, the test has been shown to discriminate between menstrual products that are not distinguished in traditional patch tests but do appear to differ in tolerability based on market surveillance [11].

Although the popliteal fossa is not intended to be a model for the vagina, this site of application and the aforementioned testing protocol offer several advantages for testing tampon materials. The popliteal fossa is quite sensitive and will respond over time to any local irritant, yet is relatively protected from external elements that could influence the inflammatory response. The site allows concurrent parallel product comparisons in the same volunteer-a significant advantage. The test design enables daily or more frequent observations over an extended period of time and allows tissue recovery to be assessed. A single-blind protocol, with examination by trained skin graders routinely involved in clinical patch tests and in-use studies, minimizes observer bias and interexaminer variability. Moreover, subsurface visualization with cross-polarized light can be incorporated at the investigator's discretion to assess subclinical effects. From a practical standpoint, the popliteal fossa is easily accessed, the procedure is non-invasive, and study participation requires little disruption to volunteers' daily routine, all of which facilitate recruitment and reduce cost and turnaround time relative to crossover product trials.

The validity and relevance of this test model for assessing tampon-related tissue irritation is supported by comparing the severity of epithelial erythema in the lower genital tract (observed colposcopically in a crossover trial of experimental and control tampons) and skin erythema scored in a BTK study with the identical (but uncompressed) tampon products (Figure 1) [12]. First, 


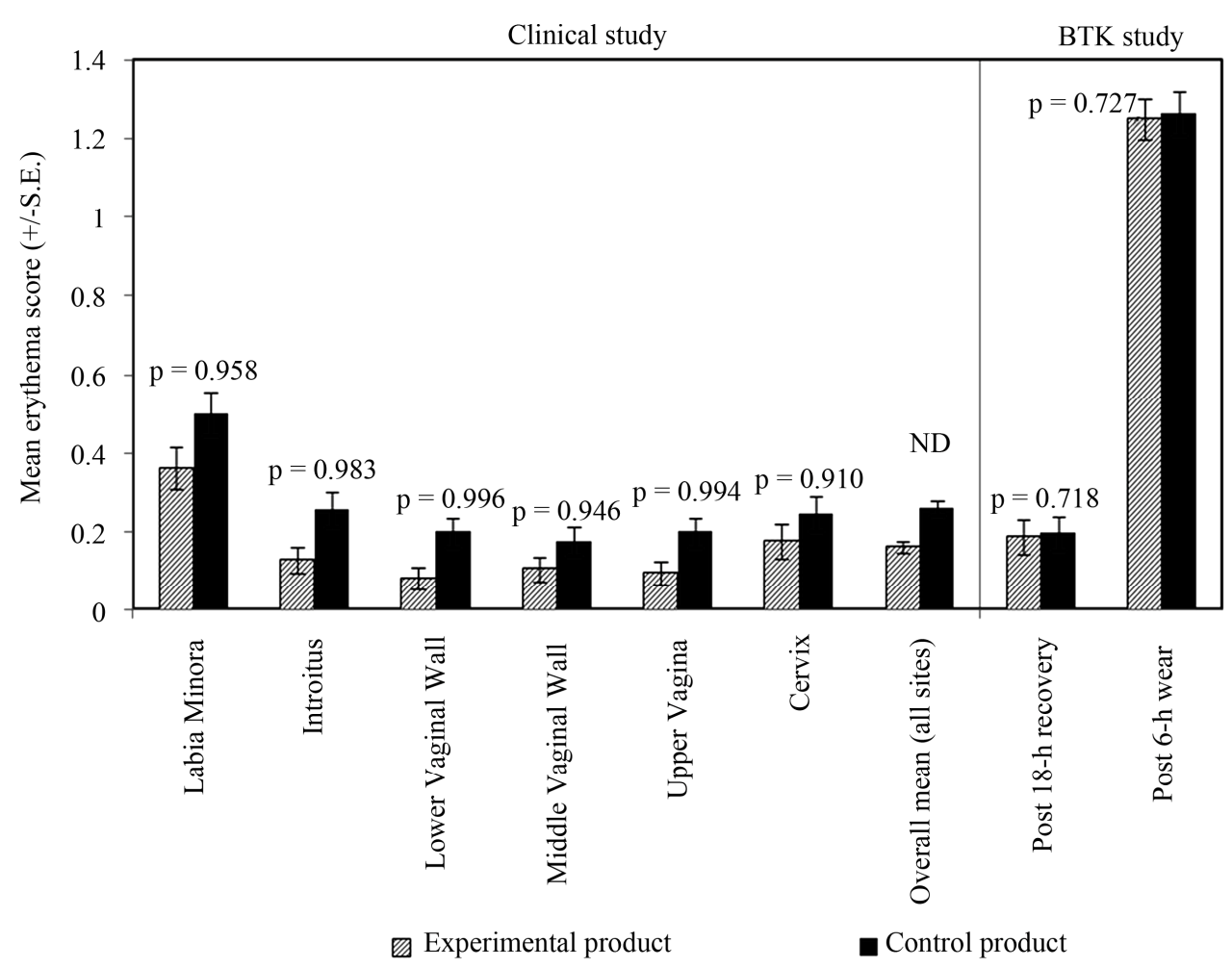

Data from Farage, M.A., Miller, K.W., Ledger, W.J. Topical Applications and the Mucosa. Curr Prob Dermatol, Vol. 40, Basel: Karger, 2011, pp. 125-132.

Figure 1. Comparison of tissue erythema from experimental and control tampons scored in the Behind-the-knee (BTK) test and following menstruation in a two-cycle crossover clinical trial.

the relative irritancy of the experimental and control products, as assessed by scoring erythema, was consistent when observed colposcopically at each site in the lower genital tract (labia minora, introitus, lower, middle and upper vagina, and cervix), and when judged by evaluating the skin of popliteal fossa either immediately after product removal (i.e. after 6 hours of wear) or after an 18-hour recovery period (Figure 1). Second, the absolute erythema scores in the BTK test after the recovery period were comparable to the overall mean scores at all genital sites combined as assessed by colposcopy in the product trial (erythema scores under both conditions were in close to 0.2 on a 5-point scale [10], where a score of 0.5 is barely discernible erythema). This is not entirely unexpected: the popliteal fossa, like the lower female genital tract, is relatively protected from the elements; moreover, intimate juxtaposition of the physical material and the epithelium occurs under both conditions and an interval of time (18 hours for the BTK or 3 to 48 hours for in-use trial) elapses between the final exposure to product and the visual assessment. Importantly, these scores also emphasize how low a level of erythema must be discerned to evaluate potential changes in minimally irritating products such as tampons, especially following recovery. Hence the value of the heightened sensitivity of the BTK protocol, evident from erythema scores dis- played immediately after product removal: these scores were approximately 6-fold higher than the scores observed after recovery (1.2 to 1.3 on a 5-point scale, where a score of 1 is faint but definite erythema and a score of 2 is moderate erythema). In short, the exaggerated exposure conditions in the BTK test improve the "signal-to-noise" ratio so that small product-related effects can be discerned more readily.

\section{CONCLUSIONS}

Enhanced visual scoring with cross-polarized light increases the sensitivity of the test even further. This was illustrated by evaluating two menstrual pads (products A and B) that consumer surveillance consistently suggested differed in tolerability yet standard skin irritation test protocols to failed to discriminate [11]. Eight of 10 comparisons in the BTK test discriminated the products based on unaided visual erythema scores, but only after repeated cumulative exposure over several days (data not shown). By contrast, subsurface visualization with crosspolarized light (but not unaided visual scoring) distinguished the irritancy potential of the two products at every evaluation point, beginning with the first exposure (Figure 2(a)) [11]. The relative frequency of subjective comments of a "burning" sensation during the test also 
discriminated the in-use tolerability of the products (Figure 2(b)). We are presently examining the potential relationships between objective scoring, subjective effects, and physical product attributes. Our preliminary research suggests that reports of "burning” or "pain", may correlate most closely with visually scored inflammation (unpublished data).

The safety assessment of intravaginal products must evaluate those endpoints most relevant to safety in-use and ensure with a high level of confidence that any observable changes in the intravaginal environment are directly product-related. Although colposcopy is part of the mandated safety assessment process for intravaginal products, lower genital tract colposcopic findings are common in healthy women and could represent the influence of confounding factors or normal physiologic changes. Colposcopic assessment has several shortcomings for assessing tampon-related effects during menstruation

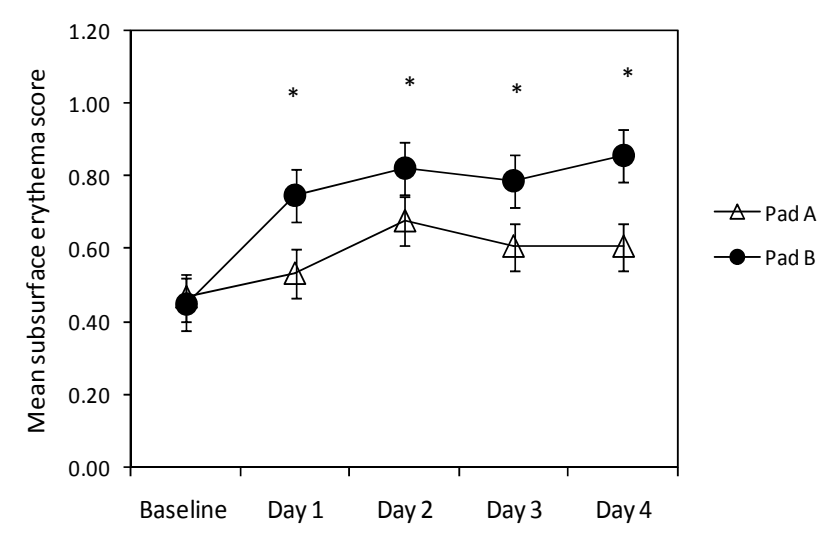

(a)

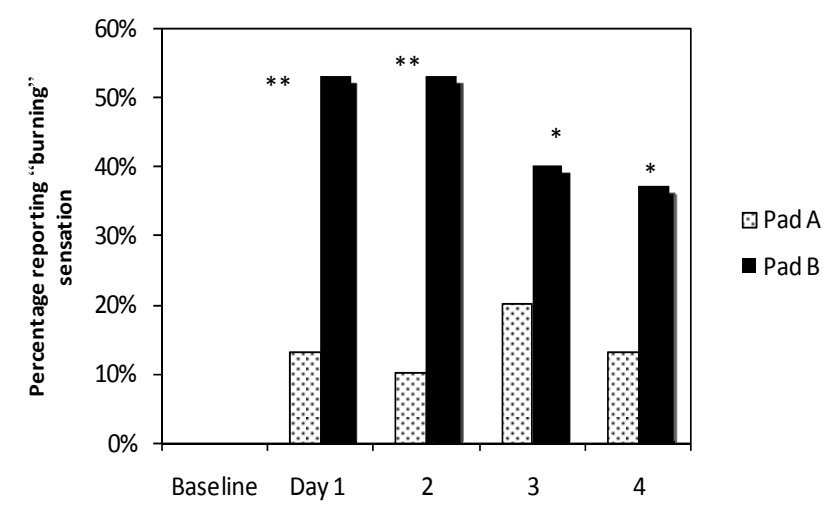

(b)

Data from Farage, M.A. Handbook of Cosmetic Science and Technology, Third ed. New York, NY: Informa Health Care, 2009, pp. 381-389.

Figure 2. Subclinical tissue erythema (mean \pm S.E.) visualized with cross-polarized light (a) and percentage of subjects reporting a burning sensation (b) associated with two commercial menstrual pads in the Behind-the-knee (BTK) test. (Pad A: open symbols; Pad B: closed symbols. ${ }^{* *}$ Significant difference between Pad A and Pad B, p $<0.001$; 'Significance difference between Pad A and Pad B, p < 0.05). and consequently relies on post-hoc evaluations of residual changes after menstrual use is complete. This commentary illustrates the advantages of the Behindthe-Knee (BTK) test, as a simple alternative for assessing tissue inflammation and dryness. The BTK test correlates well with colposcopic evaluation of mucosal inflammation, but has the advantage of allowing concurrent product comparisons and detecting inflammation and dryness with substantially higher sensitivity than either post-hoc colposcopic examination or other standard skin irritation test protocols. A notable finding is that the BTK test, coupled with enhanced visualization of subclinical erythema, discriminates between very similar products that nevertheless differ in tolerability based on market surveillance of consumer feedback, providing added confidence of its predictive value. We believe this test to be a valuable alternative to colposcopy for assessing potential tissue inflammation and dryness associated with tampons.

\section{ACKNOWLEDGEMENTS}

The authors acknowledge Deborah Hutchins, PhD ELS for assistance in preparing the manuscript.

\section{REFERENCES}

[1] CONRAD/WHO (2011) Manual for standardization of colposcopy for the evaluation of vaginal products, update 2004.

http://www.conrad.org/assets/attachments/Revised_Manu al.PDF

[2] Mauck, C.K., Baker, J.M., Birnkrant, D.B., Rowe, P.J. and Gabelnick, H.L. (2000) The use of colposcopy in assessing vaginal irritation in research. AIDS, 14, 22212227. doi:10.1097/00002030-200010200-00002

[3] O’Neill, E., Reeves, M.F. and Creinin, M.D. (2008) Baseline colposcopic findings in women entering studies on female vaginal products. Contraception, 78, 162-166. doi:10.1016/j.contraception.2008.04.002

[4] Fraser, I.S., Lahteenmaki, P., Elomaa, K., Lacarra, M., Mishell, D.R. Jr., Alvarez, F., et al. (1999) Variations in vaginal epithelial surface appearance determined by colposcopic inspection in healthy, sexually active women. Human Reproduction, 14, 1974-1978. doi:10.1093/humrep/14.8.1974

[5] Norvell, M.K., Benrubi, G.I. and Thompson, R.J. (1984) Investigation of microtrauma after sexual intercourse. The Journal of Reproductive Medicine, 29, 269-271.

[6] Ballagh, S.A., Mauck, C.K., Henry, D., Archer, D.F., Abercrombie, T., Callahan, M.M., et al. (2004) A comparison of techniques to assess cervicovaginal irritation and evaluation of the variability between two observers. Contraception, 70, 241-249.

doi:10.1016/j.contraception.2004.03.002

[7] Farage, M.A., Singh, M. and Ledger, W.J. (2009) Investigation of the sensitivity of a cross-polarized light visu- 
alization system to detect subclinical erythema and dryness in women with vulvovaginitis. American Journal of Obstetrics \& Gynecology, 201, 20e1-20e6.

[8] Wensveen, C., Kagie, M., Nagelkerke, N. and Trimbos, B. (2007) Interobserver agreement on interpreting hand drawings of colposcopy in women with borderline cytology to predict high-grade lesions. European Journal of Obstetrics, Gynecology, and Reproductive Biology, 135, 123-126. doi:10.1016/j.ejogrb.2006.08.001

[9] Cummins, J.E. Jr. and Doncel, G.F. (2009) Biomarkers of cervicovaginal inflammation for the assessment of microbicide safety. Sexually Transmitted Diseases, 36, S84S91. doi:10.1097/OLQ.0b013e3181994191

[10] Farage, M.A., Gilpin, D.A., Enane, N.A. and Baldwin, S.
(2001) Development of a new test for mechanical irritation: Behind the knee as a test site. Skin Research and Technology, 7, 193-203. doi:10.1034/j.1600-0846.2001.70309.x

[11] Farage, M.A. (2009) Sensory effects and irritation: A strong relationship. In: Barel, A.O., Paye, M. and Maibach, H.I., Eds., Handbook of Cosmetic Science and Technology, Informa Health Care, New York, 381-289.

[12] Farage, M.A., Miller, K.W. and Ledger, W.J. (2011) Can the BTK clinical test be used to evaluate the mechanical irritation potential for products intended for contact with mucous membranes? In: Surber, C., Elsner, P. and Farage, M.A., Eds., Topical Applications and the Mucosa, Karger, Basel, 125-132. doi:10.1159/000321063 